\title{
Diretriz Técnica da ANAMT (DT n0 02/2016)* - Efeito do rastreamento do uso de álcool e drogas entre trabalhadores
}

\author{
Technical Guidelines of ANAMT (DT no O2/2016) - \\ Effect of alcohol and drugs testing among workers
}

\begin{abstract}
João Silvestre Silva-Junior' ${ }^{1}$ Leandro Lessa², Daniele Pimentel Maciel'3 José Domingos Neto ${ }^{4}$, Eduardo Myung ${ }^{5}$, Fernando Akio Mariya ${ }^{6}$, Márcia Bandini 7 , Wanderley Marques Bernardo ${ }^{8}$
\end{abstract}

\section{MÉTODO DE COLETA DEEVIDÊNCIAS}

Esta diretriz seguiu padrão de uma revisão sistemática com recuperação de evidencias baseada no movimento da Medicina Baseada em Evidências (Evidence-Based Medicine), em que a experiência clínica é integrada com a capacidade de analisar criticamente e aplicar de forma racional a informação científica, melhorando assim a qualidade da assistência médica. A MBE utiliza provas científicas existentes e disponíveis no momento, com boa validade interna e externa, para a aplicação de seus resultados na prática clínica. ${ }^{1,2}$

As revisões sistemáticas são consideradas, atualmente, o nível I de evidências para qualquer questão clínica por sumarizarem sistematicamente informações sobre determinado tópico através de estudos primários (ensaios clínicos, estudos de coorte, casos-controle ou estudos transversais), utilizando-se de uma metodologia reprodutível, além de integrar informações de efetividade, eficiência, eficácia e segurança. ${ }^{1,2}$

Utilizamos a forma estruturada de formular a pergunta sintetizada pelo acrônimo P.I.C.O., onde o P corresponde ao paciente ou população, I de intervenção ou indicador, C de comparação ou controle, e $\mathrm{O}$ de "outcome" ou desfecho. A partir da pergunta estruturada identificamos as palavras-chave ou descritores que irão constituir a base da busca da evidência nas diversas bases de dados disponíveis. ${ }^{1,2}$ (Anexo I)

\section{DÚVIDA CLÍNICA}

Há repercussões sobre desfechos relacionados ao trabalho quando se realizada o rastreamento do uso de álcool e drogas entre trabalhadores?

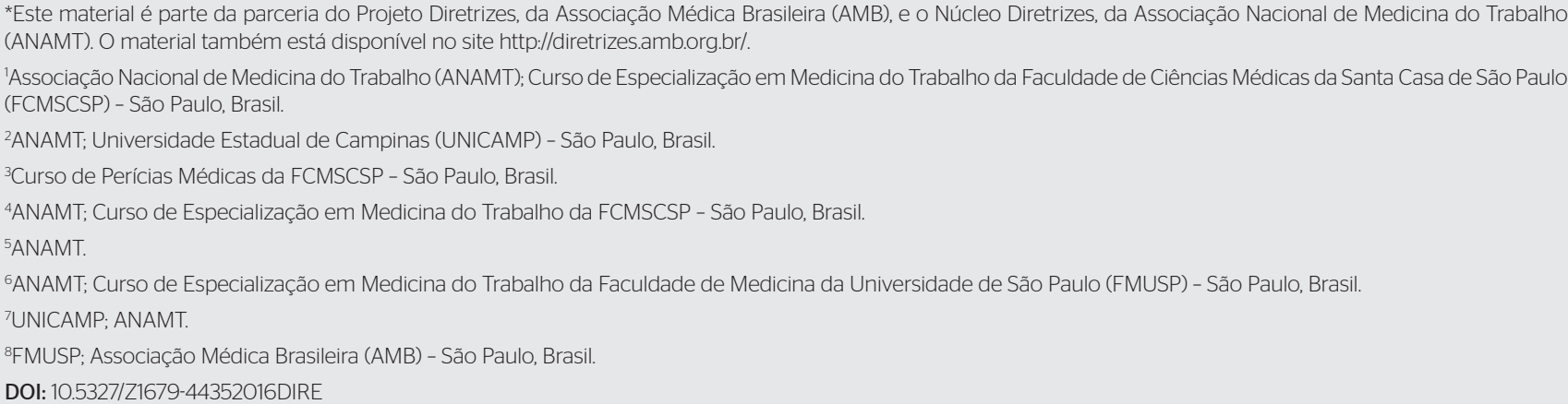




\section{GRAU DE RECOMENDAÇÃO E FORÇA DE EVIDÊNCIA}
A: Estudos experimentais ou observacionais de melhor consistência.
B: Estudos experimentais ou observacionais de menor consistência.
C: Relatos de casos / estudos não controlados.
D: Opinião desprovida de avaliação crítica, baseada em consensos, estudos fisiológicos ou modelos animais.

\section{OBJETIVO}

Esta Diretriz tem como objetivo apresentare discutira melhor evidência científica disponível na atualidade a respeito do efeito do rastreamento do uso de álcool e drogas entre trabalhadores sobre desfechos relacionados ao trabalho, como acidentes de trabalho, absenteísmo, presenteísmo e produtividade.

\section{CONFLITO DE INTERESSE}

Nenhum conflito de interesse foi declarado pelos participantes da elaboração desta diretriz.

\section{INTRODUÇÃO}

O consumo de álcool e drogas entre trabalhadores é uma realidade global e está associado a acidentes de trabalho ${ }^{3,4}$, absenteísmo ${ }^{5-7}$ e presenteísmo. ${ }^{7}$ A prevalência de testes positivos para o consumo de álcool ou drogas varia de 5\% a 15\% entre trabalhadores de diversas categorias profissionais. ${ }^{8}$ No Brasil foi detectada prevalência de 9,3\% de testes de urina positivos para anfetamina, cocaína ou canabinóides em motoristas de caminhão. ${ }^{9}$ Outro estudo demonstrou a prevalência de 3,1\% de consumo de álcool, anfetamina, cocaína ou canabinóide em testes matinais de saliva entre motoristas de caminhão. ${ }^{10}$

Desde meados de 1980 diversos países têm implantado políticas contra o uso abusivo de álcool e drogas, institucionalizando programas em serviços públicos e privados envolvendo ações como, por exemplo, a realização de testes de detecção para várias substâncias psicoativas. Nos Estados Unidos, estas políticas encontram suporte legal por meio de leis como "The Comprehensive Drug Abuse Prevention and Control Act" e o "Drug-free Workplace Act". No início do século XXI, cerca de metade das empresas americanas realizavam o rastreamento de álcool e drogas entre trabalhadores como uma ação para controle do uso abusivo. ${ }^{11}$

No processo de rastreamento, rotineiramente são realizados testes toxicológicos para detecção do histórico de consumo de álcool e drogas. Fluidos biológicos (como sangue, urina, suor, saliva) ou ar expirado são considerados matrizes para detecção de consumo recente; enquanto que cabelo e outros anexos podem indicar consumo ocorrido há meses (larga janela). Tais testes usualmente fazem parte de um programa maior de prevenção, rastreamento e tratamento do uso abusivo de álcool e drogas. ${ }^{12}$

Apesar do rastreamento do uso de álcool e drogas ser incluído como uma ação para a promoção de saúde e a prevenção de agravos, a obrigatoriedade da participação dos trabalhadores na testagem suscita questões éticas. No Brasil, o Conselho Federal de Medicina considerou em parecer ${ }^{13}$ que a solicitação de exames de monitoramento de drogas ilícitas para permitir acesso ao trabalho contraria os postulados éticos. Entretanto, é sabido que os testes toxicológicos para detecção do uso de álcool e drogas continuam sendo realizados nas rotinas dos serviços de saúde em muitas empresas brasileiras e que por vezes fazem parte do rol de exames médicos obrigatórios.

Há o exemplo do Regulamento Brasileiro da Aviação Civil no 120 (RBAC 120) ${ }^{14}$ que exige das empresas que tenham atividades de risco à segurança operacional aérea (ARSO) a implantação de um Programa de Prevenção do Risco Associado ao Uso de Substâncias Psicoativas (PPSP). Neste programa é mandatória a indicação de rastreamento de consumo de álcool e substâncias psicoativas entre trabalhadores ARSO quando em exame admissional (prévio ao início das atividades), de maneira aleatória na vigência do contrato de trabalho, após envolvimento em situação de acidente, no caso de suspeita de uso, e/ ou no retorno ou acompanhamento de usuários confirmados em testagem prévia.

Outro ponto polêmico na questão do rastreamento do uso de álcool e drogas entre trabalhadores foi a promulgação da Lei no 13.103/2015. Esta legislação alterou artigos da Consolidação das Leis do Trabalho (CLT) e legitimou a realização dos testes toxicológicos de larga janela de detecção para exames admissionais, demissionais e no intervalo médio de validade da Carteira Nacional de Habilitação $(\mathrm{CNH})$ para motoristas de transporte de carga e passageiros. ${ }^{15}$

As justificativas utilizadas para indicação do rastreamento do consumo de álcool e drogas entre trabalhadores estão relacionadas a: 
- identificação de trabalhadores que sob influência de álcool ou drogas podem apresentar risco para si ou para terceiros;

- investigação de acidentes/incidentes de trabalho;

- triagem de trabalhadores mais produtivos;

- seleção pré-admissional, para cargos de confiança ou que envolvam alto risco à vida. ${ }^{12,16}$

Neste cenário de incertezas, o médico do trabalho é cobrado pelos gestores a decidir se há indicação da testagem toxicológica entre trabalhadores sob a sua responsabilidade. Entre os supostos benefícios decorrentes desta ação, pode-se citar a redução de acidentes de trabalho, a redução do absenteísmo e/ou presenteísmo, e o aumento da produtividade do trabalhador. Em tal situação, cabem analisar os pressupostos éticos, as questões legais, as evidências científicas e o custo-benefício da implantação do rastreamento do uso de álcool e drogas entre trabalhadores.

\section{Há repercussões sobre desfechos ocupacionais (acidente de trabalho, absenteísmo, presenteísmo e/ou produtividade) quando o rastreamento do uso de álcool e drogas é realizado em trabalhadores?}

Resultados positivos de redução nos acidentes de trabalho foram descritos no estudo primário observacional ${ }^{17}$ (B) e em uma das revisões sistemáticas. ${ }^{16}$ (B) Porém, as duas revisões sistemáticas mais recentes de estudos originais não indicaram consenso do efeito positivo do rastreamento do uso de álcool e drogas sobre os acidentes de trabalho quando avaliaram dados de categorias específicas, como os motoristas profissionais, ${ }^{18}$ (A) ou trabalhadores de diversas atividades econômicas. ${ }^{19}(\mathrm{~B})$

As revisões sistemáticas consideraram a qualidade dos estudos observacionais selecionados como de moderada a fraca evidência. Os fatores que contribuíram para influenciar esta conclusão estão relacionados a questões como a metodologia utilizada (que não permite inferência de causalidade), a seleção dos participantes, o método de coleta de dados e o estudo de fatores de confusão para a compreensão da questão estudada.

Os estudos apresentaram dados relativos aos trabalhadores de diversas atividades econômicas: setor elétrico ${ }^{16}(B)$, fundição, varejo $^{19}(\mathrm{~B})$, manufatura, serviços, construção civil ${ }^{16,19}(\mathrm{~B})$, ferroviário $^{16,17,19}(\mathrm{~B})$ e transporte $^{18}(\mathrm{~A}),{ }^{16,19}(\mathrm{~B})$. Apesar da ampla gama de ocupações ser um fator positivo para a generalização dos resultados, os autores não controlaram o efeito do rastreamento de outros fatores que poderiam ter influenciado na redução dos acidentes de trabalho, entre os grupos.
Além disso, considerando a aferição do desfecho, nenhum estudo abordou a possibilidade da subnotificação de acidentes de trabalho, que poderia ser uma resposta à testagem obrigatória, principalmente nas empresas que realizam exames após acidente. Também algumas características peculiares à própria testagem, como tipo de teste ou análise independente do tipo de rastreamento (para álcool ou para drogas), comprometeram as evidências apresentadas. A frequência da testagem foi abordada por apenas um estudo a fim de apresentar parâmetros para alcançar uma maior intensidade de efeito ${ }^{17}(\mathrm{~B})$.

Para finalizar, os estudos analisados indicam que a testagem faz parte de um programa mais amplo voltado para estimular a redução do uso de álcoole drogas entre trabalhadores. Diferentes ações instituídas nos programas de prevenção do uso de álcoole drogas entre trabalhadores têm diferentes resultados quanto se avalia a repercussão na redução de consumo de álcool e/ou de drogas. ${ }^{20}$ (B) No entanto nenhum dos estudos explicitou as ações que fazem parte dos programas de prevenção de consumo de álcool e drogas implantados nas empresas. Portanto, é discutível que a redução de acidentes de trabalho, encontrada em alguns dos estudos analisados, seja efeito direto do rastreamento do uso de álcool e drogas, pois tais estudos ignoraram os possíveis efeitos da educação e orientação acerca do dano à saúde, da assistência à saúde, entre outras ações de um programa amplo.

Sobre os desfechos absenteísmo, presenteísmo e produtividade, não foram encontradas evidências científicas de qualquer efeito a partir da implantação do rastreamento do uso de álcool e drogas isoladamente ou como parte de um programa em empresas.

\section{RECOMENDAÇÃO}

O consolidado de informações apresentadas pelos estudos triados indica não haver evidência suficiente para a recomendação de rastreamento de uso de álcool e drogas entre trabalhadores como medida isolada para a redução de acidente de trabalho nas empresas.

Não há evidências atuais na literatura cientifica suficientes para avaliar o equilíbrio entre benefícios e malefícios em relação à realização do rastreamento para o uso de álcool e drogas entre trabalhadores como medida isolada para redução de acidentes de trabalho.

Não recomendamos a aplicação do rastreamento por exame toxicológico, em janela curta ou larga, na prática da Medicina do Trabalho. 


\section{REFERÊNCIAS}

1. Nobre MR, Bernardo WM, Jatene FB. A prática clínica baseada em evidencias. Parte I- Questões clínicas bem construídas. Rev Assoc Med Bras 2003; 49(4):445-9.

2. Bernardo WM, Nobre MR, Jatene FB. A prática clínica baseada em evidencias. Parte II - Questões clínicas bem construídas. Rev Assoc Med Bras 2004; 50(1):104-8.

3. Spicer RS, Miller TR, Smith GS. Worker substance use, workplace problems and the risk of occupational injury: A matched casecontrol study. Journal of Studies on Alcohol. 2003;64(4)111:570-578.

4. Li Y, Bai Y. Comparison of characteristics between fatal and injury acidentes in the highway construction zones. Saf. Sci. 2008;46(4):646-660.

5. Bass AR et al. Employee drug use, demographic characteristics, work reactions, and absenteeism. Journal of Occupational Health Psychology. 1996;1(1):92-99.

6. Pidd KJ, Berry JG, Roche AM, Harrison JE. Estimating the cost of alcohol-related absenteeism in the Australian workforce: The importance of consumption patterns. Medical Journal of Australia. 2006;185(11-12):637-641.

7. de Graff R, Tuithof M, van Dorsselaer S, ten Have M. Comparing the effects on work performance of mental and physical disorders. Social Psychiatry and Psychiatric Epidemiology. 2012;47:1873-1883.

8. Zwerling C. Current practice and experience in drug and alcohol testing in the workplace. Bull Narc. 1993;45: 155-96.

9. Leyton $\mathrm{V}$ et al. Amphetamine, cocaine and cannabinoids use among truck drivers on the roads in the State of Sao Paulo, Brazil. Forensic science international. 2012;215(1):25-27.

10. Yonamine M, Sanches LR, Bismara Paranhos BAP, Almeida RM, Andreuccetti G, Leyton V. Detecting alcohol and illicit drugs in oral fluid samples collected from truck drivers in the state of São Paulo, Brazil. Traffic injury prevention. 2013;14(2):127-131.

11. Larson SL, Eyerman J, Foster MS, Gfroerer JC. Worker Substance Use and Workplace Policies and Programs. Substance Abuse and Mental Health Services Administration (SAMHSA). Rockville, MD: Office of Applied Statistics, 2007.

12. Morland J. Types of drug-testing programs in the workplace. Bull Narc. 1993;45:83-113.

13. Conselho Federal de Medicina (CFM). Parecer CFM no 26. Monitoramento de drogas ilícitas em urina e sangue, para permitir acesso ao trabalho. Brasília: CFM, 2012.

14. Brasil. Agência Nacional de Aviação Civil. Regulamento Brasileiro de Aviação Civil (RBAC) no 120. Programa De Prevenção Do Risco Associado Ao Uso Indevido De Substâncias Psicoativas Na Aviação Civil. Brasília: Diário Oficial da União, 31 mai 2011.

15. Brasil. Presidência da República. Lei no 13.103. Dispõe sobre o exercício da profissão de motorista; altera a Consolidação das Leis do Trabalho - CLT, aprovada pelo Decreto-Lei no 5.452, de 1o de maio de 1943, e as Leis nos 9.503, de 23 de setembro de 1997 - Código de Trânsito Brasileiro, e 11.442, de 5 de janeiro de 2007 (empresas e transportadores autônomos de carga), para disciplinar a jornada de trabalho e o tempo de direção do motorista profissional; altera a Lei no 7.408 , de 25 de novembro de 1985; revoga dispositivos da Lei no 12.619, de 30 de abril de 2012; e dá outras providências. Brasília: Diário Oficial da União, 03 mar 2015.

16. Kraus JF. The effects of certain drug-testing programs on injury reduction in the workplace: an evidence-based review. International journal of occupational and environmental health. 2001;7(2):103-108.

17. Marques PH, Jesus V, Olea SA, Vairinhos V, Jacinto C. The effect of alcohol and drug testing at the workplace on individual's occupational accident risk. Safety science. 2014;68:108-120.

18. Cashman CM, Ruotsalainen JH, Greiner BA, Beirne PV, Verbeek JH. Alcohol and drug screening of occupational drivers for preventing injury. Cochrane Database Syst. Rev. 2009;(2):CD006566.

19. Pidd $\mathrm{K}$, Roche AM. How effective is drug testing as a workplace safety strategy? A systematic review of the evidence. Accident Analysis \& Prevention. 2014;71:154-165.

20. Pidd K, Kostadinov V, Roche A. Do workplace policies work? An examination of the relationship between AOD policies and workers' substance use. International Journal of Drug Policy. 2016;28:48-54.

21. Lange WR, Cabanilla BR, Moler G, Bernacki EJ, Frankenfie DL, Fudala PJ. Preemployment drug screening at the Johns-Hopkins Hospital, 1989 and 1991. Am. J. Drug Alcohol Abuse. 1994;20(1):35-46.

22. Gerber JK, Yacoubian GS. Evaluation of drug testing in the workplace: studyof the construction industry. J. Constr. Eng. M. Asce. 2001;127 (6):438-444.

23. Miller TR, Zaloshnja E, Spicer RS. Effectiveness and benefit-cost of peer-based workplace substance abuse prevention coupled with random testing. Accid. Anal. Prev. 207;39(3):565-573.

24. Brady JE, Baker SP, DiMaggio C, McCarthy ML, Rebok GW, Li G. Effectiveness of mandatory alcohol testing programs in reducing alcohol involvmentin fatal motor carrier crashes. Am. J. Epidemiol. 2009;170(6):775-782.

25. Schofield KE, Alexander BH, Gerberich SG, Ryan AD. Injury rates, sever-ity, and drug testing programs in small construction companies. J. Saf. Res. 2013;44:97-104.

26. Feinauer DM, Havlovic SJ. Drug testing as a strategy to reduce occupationalaccidents: a longitudinal analysis. J. Saf. Res. 1993;24(1):1-7.

27. Wickizer TM, Kopjar B, Franklin G, Joesch J. Do drug-free workplace pro-grams prevent occupational injuries? Evidence from Washington State. HealthServ. Res. 2004;39:91-110.

28. Effective Public Health Practice Project, 1998. Quality Assessment Tool For Quanti-tative Studies, Retrieved January 2013, from http:// www.ephpp.ca/index.html

29. Jadad AR, Moore RA, Carroll D, Jenkinson C, Reynolds DJ, Gavaghan DJ, et al. Assessing the quality of reports of randomized clinical trials: is blinding necessary? Control Clin Trials 1996; 17:1-12.

30. Goldet G, Howick J. Understanding GRADE: an introduction. J Evid Based Med 2013; 6:50-4.

31. Wells G, Shea B, O'Connell D, Robertson J, Peterson J, Welch V, et al. The Newcastle-Ottawa Scale (NOS) for assessing the quality of nonrandomised studies in meta-analyses.

32. Levels of Evidence and Grades of Recommendations - Oxford Centre for Evidence Based Medicine. Disponível em URL: http:// cebm.jr2.ox.ac.uk/docs/old_levels.htm. 


\section{ANEXO I}

\section{DÚVIDA CLÍNICA}

Há repercussões sobre desfechos relacionados ao trabalho quando se realizada o rastreamento do uso de álcool e drogas entre trabalhadores?

\section{PERGUNTA ESTRUTURADA}

P: Trabalhadores

I: Rastreamento do uso de álcool e drogas

C: Não rastrear o uso de álcool e drogas

O: Desfechos relacionados ao trabalho (acidente de trabalho, absenteísmo, presenteísmo, alteração na produtividade)

\section{ESTRATÉGIA DE BUSCA DE EVIDÊNCIA}

\subsection{MEDLINE/PUBMED:}

(( (screening OR substance abuse detection OR drug testing) AND (cannabi* OR opia* OR cocaine OR amphetamine* OR marijuana OR illicit drug* OR street drug* OR alcohol OR drink* OR binge) AND (work* OR occupational $\left.^{*}\right)$ ) AND (diagnosis/broad[filter] OR therapy/ broad[filter] OR prognosis/broad[filter]))

\subsection{COCHRANE CENTRAL,} PSYCINFO, EBSCO/CINAHL, LILACS:

((screening ORsubstance abuse detection OR drug testing) AND (cannabi* OR opia* OR cocaine OR amphetamine* OR marijuana OR illicit drug* OR street drug* OR alcohol OR drink* OR binge) AND (work* OR occupational ${ }^{*}$ ))

\section{TRABALHOS RECUPERADOS}

A obtenção da evidência a ser utilizada para análise da questão clínica seguiu as etapas de: elaboração da questão clínica, estruturação da pergunta, busca da evidência, avaliação crítica e seleção da evidência, exposição dos resultados e recomendações.
A partir da pergunta norteadora foram discutidos, pesquisados e definidos os descritores a partir do Medical Subject Headings (MESH) e dos Descritores em Ciência de Saúde (DeCS). Na sequência foram construídas estratégias de busca específicas para as bases de dados a serem consultadas: MEDLINE via PubMed, Cochrane Central, PsycINFO, Ebsco/CINAHL e LILACS.

Foram recuperados 7.634 resultados, até 07/05/2016 (Tabela 1). Tais resultados foram triados independentemente por dois pesquisadores que levaram em consideração se o título e resumo do trabalho abrangiam o escopo proposto para esta Diretriz. Os estudos que não tiveram dupla concordância dos avaliadores foram arbitrados por um terceiro avaliador a fim de definir para inclusão ou exclusão do estudo na revisão. Ao final desta etapa, 74 trabalhos foram selecionados para leitura do artigo completo.

Após o processo de leitura dos artigos completos, houve a seleção de sete referências: cinco artigos originais ${ }^{21-25} \mathrm{e}$ duas revisões sistemáticas ${ }^{18,19}$. A busca na literatura cinzenta acrescentou mais três artigos originais ${ }^{17,26,27}$ e uma revisão sistemática ${ }^{16}$.

Uma das revisões sistemáticas ${ }^{19}$ consolidou a evidência de sete dos oito estudos originais triados. $\mathrm{O}$ critério utilizado pelos autores para a avaliação metodológica foi Effective Public Health Practice Project (EPHPP), que sistematiza a qualidade de estudos primários em saúde pública. ${ }^{28}$ Para tentar manter homogeneidade de avaliação, este método também foi utilizado pelos autores da Diretriz para classificar a única referência com dados primários que não fazia parte de revisão sistemática. ${ }^{17}$

Tabela 1. Distribuição absoluta e relativa dos resultados da busca de referências conforme banco de dados acessados.

\begin{tabular}{l|c|c} 
Banco de dados & $\mathrm{n}$ & $\%$ \\
\hline PubMed/MEDLINE & 6815 & 89,3 \\
\hline Ebsco/CINAHL & 460 & 6,0 \\
\hline Cochrane Central & 205 & 2,7 \\
\hline PsycINFO & 130 & 1,7 \\
\hline LILACS & 24 & 0,3 \\
\hline Total & 7634 & \\
\hline
\end{tabular}


Ao final do processo metodológico, restaram três revisões sistemáticas de estudos observacionais ${ }^{16,18,19}$ e um estudo observacional de coorte ${ }^{17}$ para análise da evidência.

\section{CRITÉRIOS DE INCLUSÃOE EXCLUSÃO DOS TRABALHOS}

Para serem incluídos na etapa de análise da evidência os estudos deveriam avaliar um ou mais desfechos ligados ao trabalho de interesse da Diretriz (acidente de trabalho, absenteísmo, presenteísmo, produtividade). Também era necessário que na sessão de métodos fosse indicado a realização da intervenção de interesse, isto é, o rastreamento de álcool e/ou drogas entre trabalhadores objeto do estudo.

\subsection{SEGUNDO OS DESENHOS DE ESTUDO}

Não foram considerados monografias ou resumos de apresentação em evento científico. Os estudos deveriam ser revisão (sistemática ou metanálise) ou trabalho de campo (observacional ou experimental) que apresentassem evidência científica. Revisões sistemáticas e meta-análises foram utilizadas com o princípio de recuperação de referências que porventura haviam sido perdidas em primeiro momento a partir da estratégia de busca inicial. Os ensaios clínicos controlados foram avaliados segundo o escore $\mathrm{JADAD}^{27}$ e o escore GRADE ${ }^{3}$.

\subsection{IDIOMA}

Foram incluídos estudos disponíveis na língua portuguesa, inglesa ou espanhola.

\subsection{SEGUNDO A PUBLICAÇÃO}

Somente os trabalhos cujos textos completos se encontravam disponíveis foram considerados para avaliação crítica.

\section{MÉTODO DE AVALIAÇÃO CRÍTICA}

Quando, após a aplicação dos critérios de inclusão e exclusão, a evidência selecionada foi definida como ensaio clínico controlado randomizado (ECR), era submetida a um Check-list apropriado de avaliação crítica (Tabela 2). A avaliação crítica do ECR permite classificá-lo segundo o escore JADAD ${ }^{29}$, considerando os ensaios JADAD $<$ três (3) como inconsistentes (grau $\mathrm{B}$ ), e aqueles com escore $\geq$ três (3), consistentes (grau A), e segundo o escore GRADE G $^{30}$ (evidência forte ou moderada).

Quando a evidência selecionada foi definida como estudo comparativo (coortes observacionais ou ensaio clínico não randômico), esta era submetida a um Check-list apropriado de avaliação crítica (Tabela 3), permitindo a classificação do estudo, segundo o escore NEW CASTLE OTAWA SCALE ${ }^{31}$, considerando os estudos coortes consistentes com escore $\geq 6$ e inconsistentes $<6$.

Tabela 2. Roteiro de avaliação crítica de ensaios clínicos controlados randomizados.

\begin{tabular}{|c|c|}
\hline $\begin{array}{l}\text { Dados do estudo } \\
\text { Referência, Desenho de } \\
\text { estudo, JADAD, força da } \\
\text { evidência }\end{array}$ & $\begin{array}{c}\text { Cálculo da amostra } \\
\text { Diferenças estimadas, } \\
\text { poder, nível de significância, } \\
\text { total de pacientes }\end{array}$ \\
\hline $\begin{array}{l}\text { Seleção dos pacientes } \\
\text { Critérios de inclusão } \\
\text { e exclusão }\end{array}$ & $\begin{array}{c}\text { Pacientes } \\
\text { Recrutados, randomizados, } \\
\text { diferenças prognósticas }\end{array}$ \\
\hline $\begin{array}{c}\text { Randomização } \\
\text { Descrição e alocação } \\
\text { vendada }\end{array}$ & $\begin{array}{l}\text { Seguimento dos pacientes } \\
\text { Tempo, perdas, migração }\end{array}$ \\
\hline $\begin{array}{l}\text { Protocolo de tratamento } \\
\text { Intervenção, controle } \\
\text { e cegamento }\end{array}$ & $\begin{array}{c}\text { Análise } \\
\text { Intenção de tratamento, anali- } \\
\text { sados intervenção e controle }\end{array}$ \\
\hline $\begin{array}{l}\text { Desfechos considerados } \\
\text { Principal, secundário, instru- } \\
\text { mento de medida do desfe- } \\
\text { cho de interesse }\end{array}$ & $\begin{array}{c}\text { Resultado } \\
\text { Benefício ou dano em } \\
\text { dados absolutos, benefício } \\
\text { ou dano em média }\end{array}$ \\
\hline
\end{tabular}

Tabela 3. Roteiro de avaliação crítica de estudos coortes.

\begin{tabular}{|c|c|c|c|c|c|c|}
\hline $\begin{array}{l}\text { Representati- } \\
\text { vidade dos } \\
\text { expostos e sele- } \\
\text { ção dos não } \\
\text { expostos } \\
\text { (máx. } 2 \text { pontos) }\end{array}$ & $\begin{array}{c}\text { Definição da } \\
\text { exposição } \\
\text { (máx. } 1 \text { ponto) }\end{array}$ & $\begin{array}{l}\text { Demonstração de } \\
\text { que o desfecho } \\
\text { de interesse não } \\
\text { estava presente no } \\
\text { início do estudo } \\
\text { (máx. 1 ponto) }\end{array}$ & $\begin{array}{c}\text { Comparabili- } \\
\text { dade na base } \\
\text { do desenho ou } \\
\text { da análise } \\
\text { (máx. } 2 \text { pontos) }\end{array}$ & $\begin{array}{c}\text { Avaliação do } \\
\text { desfecho } \\
\text { (máx. } 1 \text { ponto) }\end{array}$ & $\begin{array}{c}\text { Tempo apro- } \\
\text { priado de } \\
\text { seguimento } \\
\text { (máx. } 2 \text { pontos) }\end{array}$ & $\begin{array}{c}\text { Escore e } \\
\text { nível da } \\
\text { evidência }\end{array}$ \\
\hline
\end{tabular}




\section{EXPOSIÇÃO DOS RESULTADOS}

Para resultados com evidência disponível serão definidos de maneira específica, sempre que possível, a população, a intervenção, os desfechos, a presença ou ausência de benefício e/ou dano e as controvérsias.

Os resultados serão expostos preferencialmente em dados absolutos, risco absoluto, número necessário para tratar (NNT), ou número para produzir dano (NNH), e eventualmente em média e desvio padrão (tabela 4 ).

\section{RECOMENDAÇÕES}

As recomendações serão elaboradas pelos autores da revisão, com a característica inicial de síntese da evidência,
Tabela 4. Planilha utilizada para descrição e exposição dos resultados de cada estudo.

\begin{tabular}{c}
\hline Evidência incluída \\
Desenho do estudo \\
\hline População selecionada \\
\hline Tempo de seguimento \\
Desfechos considerados \\
\hline Expressão dos resultados: porcentagem, risco, odds, \\
hazard ratio, média
\end{tabular}

sendo submetida a validação por todos os autores participantes da elaboração da Diretriz.

O grau de recomendação a ser utilizado advém diretamente da força disponível dos estudos incluídos segundo Oxford $^{32}$, e da utilização do sistema GRADE ${ }^{30}$. 